\title{
AN APPROXIMATE MINIMUM MOSPA ESTIMATOR
}

\author{
David Frederic Crouse ${ }^{\dagger}$, Peter Willett ${ }^{\dagger}$, Marco Guerriero $\ddagger$ and Lennart Svensson* \\ † University of Connecticut, 371 Fairfield Way, U-2157, Storrs, CT 06269, USA \\ $\ddagger$ Elt Elettronica S.p.A., Via Tiburtina Valeria Km 13, 700 - 00131 Roma, Italy \\ *Department of Signals and Systems, Chalmers University of Technology, SE-412 96 Göteborg, Sweden
}

\begin{abstract}
Optimizing over a variant of the Mean Optimal Subpattern Assignment (MOSPA) metric is equivalent to optimizing over the track accuracy statistic often used in target tracking benchmarks. Past work has shown how obtaining a Minimum MOSPA (MMOSPA) estimate for target locations from a Probability Density Function (PDF) outperforms more traditional methods (e.g. maximum likelihood (ML) or Minimum Mean Squared Error (MMSE) estimates) with regard to track accuracy metrics. In this paper, we derive an approximation to the MMOSPA estimator in the two-target case, which is generally very complicated, based on minimizing a Bhattacharyya-like bound. It has a particularly nice form for Gaussian mixtures. We thence compare the new estimator to that obtained from using the MMSE and the optimal MMOSPA estimators.
\end{abstract}

Index Terms - MOSPA, tracking, state estimation

\section{INTRODUCTION}

What is it that one wishes to get from a target tracking algorithm? Based upon criteria often used in tracking benchmarks, at a particular time one wants to know where there are targets, and which targets correspond to which locations. However, many tracking algorithms do not perform optimization over the correct quantities to answer these questions in an "optimal" way.

Common criterion for deciding "where the targets are now" is to take the mean of the most likely hypothesis, or to optimize over the Mean Squared Error (MSE) of the target states [2]. However, the most likely hypothesis doesn't take into account additional uncertainty from other hypotheses, and, as discussed in [3], the MMSE estimate can lead to track coalescence when a large degree of uncertainty in the track identities exists. Optimizing over the MOSPA metric [7] would perhaps be more desirable. In one form, this is the same as finding the MMSE estimate, except the assignment of tracks to truth is not specified. This is equivalent to optimizing over the track accuracy statistic commonly used in tracking benchmarks [4]. Thus, a MOSPA-optimal algorithm should outperform other algorithms in common benchmarks.

This work was partially supported by the Office of Naval Research under contracts N00014-09-10613 and N00014-10-10412.
In Section 2 we discuss the MOSPA Statistic and the MMOSPA estimator, which was originally introduced in [5].

In this paper, we concern ourself with approximating the MMOSPA estimate for two targets when given a representation of the target PDF. ${ }^{1}$ In Section 2 we review the OSPA metric and the problem of optimizing over the mean of this metric. Due to the complexity of said problem, in Section 3 we consider an approximation to the MMOSPA estimate. The approximation may be used easily with any PDF whose moment generating function is explicit. In Section 4 we demonstrate the use of the approximation on Gaussian mixture distributions. The results are summarized in Section 5.

\section{THE MOSPA STATISTIC AND THE MMOSPA ESTIMATE}

The Optimal Subpattern Assignment (OSPA) metric, discussed in detail in [7], is given in its most general form in (1). It is used to compare a vector of state estimates for $T$ tracks, $\hat{\mathbf{x}}$, to a vector of the $N_{T}$ true target locations $\mathbf{x}$. Each of these vectors represents the stacked set of states for each track or target $^{2}$. Thus

$$
\mathbf{x}=\left[x_{1}^{T}, x_{2}^{T}, \ldots, x_{N_{T}}^{T}\right]^{T}
$$

where the superscript of $T$ represents a transpose and $x_{i}$ is the state of target $i$. The variable $p$ in (1) is an arbitrary number larger than one. The distance metric $d^{(c)}$ is defined to be

$$
d^{(c)}\left(\hat{x}_{a(t)}, x\right)=\min \left[c, d\left(\hat{x}_{a(t)}, x_{t}\right)\right]
$$

where $d$ is an arbitrary distance metric. We shall use the $n$th power of the $l_{2}$ norm, $d\left(\hat{x}_{a(t)}, x_{t}\right)=\left\|\hat{x}_{a(t)}-x_{t}\right\|^{n}$. The value $c$ is a cutoff for the maximum allowable error added by a single track. The vector $\mathbf{a}$ is a permutation vector consisting of components

$$
\mathbf{a}=\left[a_{1}, a_{2}, \ldots, a_{\min \left[T, N_{T}\right]}\right]^{T}
$$

It assigns a permutation of the targets to the ordered tracks. All together, OSPA is a very general metric for measuring

\footnotetext{
${ }^{1}$ In this paper, we consider only estimates for display at a particular time the PDF could come from any tracker, e.g. the MHT or a particle filter.

${ }^{2}$ A target is the "truth"; a track is what a tracker thinks is a target.
} 


$$
\bar{d}^{(c)}(\hat{\mathbf{x}}, \mathbf{x}) \triangleq \begin{cases}\left(\frac{1}{T} \min _{\mathbf{a}} \sum_{t=1}^{N_{T}} d^{(c)}\left(\hat{x}_{t}, x_{a(t)}\right)^{p}+c^{p}\left(T-N_{T}\right)\right)^{1 / p} & \text { if } N_{T} \leq T . \\ \left(\frac{1}{N_{T}} \min _{\mathbf{a}} \sum_{t=1}^{T} d^{(c)}\left(\hat{x}_{a(t)}, x_{t}\right)^{p}+c^{p}\left(N_{T}-T\right)\right)^{1 / p} & \text { if } N_{T}>T\end{cases}
$$

an "orderless" distance between a set of tracks and targets, penalizing a mismatch in the number of tracks and targets by approximately $c$, and capping the error contribution from any track by approximately $c$.

In this paper, we shall consider a specific form of the OSPA metric in the two target case, namely, that when the number of tracks and targets are equal $T=N_{T}=2, p=1$, and we take the limit of $c \rightarrow \infty$ and we use a norm as the distance metric, $d$. Taking the expected value over the PDF of $\mathrm{x}$, we get a MOSPA statistic for two targets

$$
\mathrm{E}\left[\bar{d}^{(c)}(\hat{\mathbf{x}}, \mathbf{x})\right]=\frac{1}{N_{T}} \mathrm{E}\left[\min \left\{\left\|\left[\begin{array}{c}
\hat{x}_{1} \\
\hat{x}_{2}
\end{array}\right]-\mathbf{x}\right\|^{n}\left\|\left[\begin{array}{c}
\hat{x}_{2} \\
\hat{x}_{1}
\end{array}\right]-\mathbf{x}\right\|^{n}\right\}\right]
$$

The MMOSPA estimate optimizes over $\hat{\mathbf{x}}$ in (5). Note that the MMOSPA estimate of a given PDF is generally neither the mean nor the most likely point of the PDF. It was derived in [5], and has an integral form that, in general, can not be explicitly solved. In the following section, we shall derive a simple approximation for this optimization.

Though minimizing the MOSPA statistic tells us where targets are located, it says nothing about which locations correspond to which targets. However, this can be solved in a second optimization step, if desired. Given a set of position estimates, and the PDFs of the targets, we can calculate something similar to an association matrix, $A=\left[a_{i, j}\right]$, where $a_{i, j}$ is the probability that estimate $i$ is closer to the true location of target $j$ than to any other target. The most likely identities of the target estimates can then be determined using the $\mathrm{JVC}^{3}$ or auction algorithms [6].

\section{AN APPROXIMATION OF THE MMOSPA ESTIMATOR}

We shall consider the case where $n=4$ in $(5)^{4}$. The MOSPA error metric from (5) for the special case of two targets may be expressed and bounded as follows ${ }^{5}$

$$
\begin{aligned}
& \frac{1}{N_{T}} \int_{\mathbf{x} \in \mathbb{R}^{2 d}} \min \left[\left\|\mathbf{x}-\hat{\mathbf{x}}_{1}\right\|^{n},\left\|\mathbf{x}-\hat{\mathbf{x}}_{2}\right\|^{n}\right] p(\mathbf{x}) d \mathbf{x} \\
& \leq \frac{1}{N_{T}} \int_{\mathbf{x} \in \mathbb{R}^{2 d}}\left\|\mathbf{x}-\hat{\mathbf{x}}_{1}\right\|^{n \beta}\left\|\mathbf{x}-\hat{\mathbf{x}}_{2}\right\|^{n(1-\beta)} p(\mathbf{x}) d \mathbf{x}
\end{aligned}
$$

\footnotetext{
${ }^{3}$ Joncker-Volgenant-Castanon.

${ }^{4} \mathrm{~A}$ similar closed-form solution can be found for any even value of $n>2$. We chose $n=4$, because it has the simplest form

${ }^{5}$ Proof: Suppose that $a \geq b \geq 0$. We know that if $0 \leq \beta \leq 1$ then $\left(\frac{a}{b}\right)^{\beta} \geq 1$. Thus, $a^{\beta} b^{1-\beta} \geq \bar{b}$. Subsequently, $\min [a, b] \leq a^{\beta} b^{1-\beta}$, because we know that $a \geq b$. We arrive at the same conclusion if $b \geq a$.
}

where $d$ is the dimensionality of the state and $p(\mathbf{x})$ is the PDF of the targets. $\hat{\mathbf{x}}_{2}$ is the same as $\hat{\mathbf{x}}_{1}$, but with the ordering of the targets reversed. $\hat{\mathbf{x}}_{2}$ may also be expressed as $\hat{\mathbf{x}}_{2}=\chi \hat{\mathbf{x}}_{1}$ where

$$
\chi \triangleq\left[\begin{array}{ll}
\mathbf{0}_{d} & \mathbf{I}_{d} \\
\mathbf{I}_{d} & \mathbf{0}_{d}
\end{array}\right]
$$

and $\mathbf{I}_{d}$ and $\mathbf{0}$ are $d$-dimensional identity and zero matrices.

To get the MMOSPA estimate we would minimize the left-hand side of (6) over $\hat{\mathrm{x}}$. However, in order to simplify things and eliminate the integral, we are going to optimize the right-hand side of (6) over $\hat{\mathbf{x}}$. The rest of this suggestion shall describe how to simplify the right-hand side of (6), and how to derive the gradient and Hessian needed to perform numeric optimization.

The inequality in (6) is based on the same relation that forms the basis of the Chernoff and Bhattacharyya bounds. If we say that $\beta=1 / 2$, as in the Bhattacharyya bound, and $n=$ 4 , i.e. we are using the $l_{2}$ norm raised to the fourth power, then if we can calculate moments of the multivariate distribution of $x$, we can eliminate all of the integration. Noting that $\hat{\mathbf{x}}_{1}^{T} \hat{\mathbf{x}}_{1}=$ $\hat{\mathbf{x}}_{2}^{T} \hat{\mathbf{x}}_{2}$, evaluating the integral with $\beta=1 / 2$ and $n=4$, our optimization problem becomes

$$
\begin{aligned}
& \min _{\hat{\mathbf{x}}} \frac{1}{N_{T}} \int_{\mathbf{x} \in \mathbb{R}^{2 d}}\left\|\mathbf{x}-\hat{\mathbf{x}}_{1}\right\|^{2}\left\|\mathbf{x}-\hat{\mathbf{x}}_{2}\right\|^{2} p(\mathbf{x}) d \mathbf{x} \\
= & \min _{\hat{\mathbf{x}}} \frac{1}{N_{T}} \mathrm{E}\left[\left(\mathbf{x}^{T} \mathbf{x}\right)^{2}-2\left(\mathbf{x}^{T} \mathbf{x}\right) \mathbf{x}^{T} A \hat{\mathbf{x}}_{1}+2\left(\mathbf{x}^{T} \mathbf{x}\right)\left(\hat{\mathbf{x}}_{1}^{T} \hat{\mathbf{x}}_{1}\right)\right. \\
& \left.-2\left(\hat{\mathbf{x}}_{1}^{T} \hat{\mathbf{x}}_{1}\right)\left(\mathbf{x}^{T} A \hat{\mathbf{x}}_{1}\right)+4\left(\mathbf{x}^{T} \hat{\mathbf{x}}_{1}\right)\left(\mathbf{x}^{T} \chi \hat{\mathbf{x}}_{1}\right)+\left(\hat{\mathbf{x}}_{1}^{T} \hat{\mathbf{x}}_{1}\right)^{2}\right]
\end{aligned}
$$

$A \triangleq \chi+\mathbf{I}_{2 d}$

\subsection{The Solution for a Multivariate Gaussian Distribu- tion}

If $p(\mathbf{x})$ is a multivariate Gaussian distribution with mean $\mu$ and covariance matrix $P$ we can easily simplify (9). We shall note the following noncentral moments of the multivariate normal distribution ${ }^{6}$

$$
\begin{aligned}
\mathrm{E}\left[\mathbf{x}^{T} \mathbf{c}\right] & =\mu^{T} \mathbf{c} \\
\mathrm{E}\left[\mathbf{x}^{T} \mathbf{x}\right] & =\operatorname{tr}[P]+\mu^{T} \mu \\
\mathrm{E}\left[\left(\mathbf{x}^{T} \mathbf{c}_{1}\right)\left(\mathbf{x}^{T} \mathbf{c}_{2}\right)\right] & =\mathbf{c}_{1}^{T}\left(P+\mu \mu^{T}\right) \mathbf{c}_{2}
\end{aligned}
$$

\footnotetext{
${ }^{6}$ These quantities were found using the method described in [1], which can be used for finding similar moments of any multivariate PDF whose moment generating function is explicit.
} 


\begin{tabular}{|c|cccc|cccc|}
\hline \multirow{2}{*}{ Example } & \multicolumn{4}{|c|}{ MOSPA, $n=2$} & \multicolumn{4}{c|}{ MOSPA, $n=4$} \\
& $\mu$ & $\mu_{M L}$ & $\mu_{4}^{B}$ & $\mu_{2}^{\text {opt }}$ & $\mu$ & $\mu_{M L}$ & $\mu_{4}^{B}$ & $\mu_{4}^{\text {opt }}$ \\
\hline Two Gaussian Mixture & 6.55 & 3.25 & 3.20 & 3.01 & 231 & 65.4 & 41.5 & 41.4 \\
Six Gaussian Mixture & 0.626 & 0.597 & 0.351 & 0.333 & 1.08 & 1.31 & 0.364 & 0.336 \\
\hline
\end{tabular}

Table 1. The results of the simulations out to three significant digits. The MOSPA error for $n=4$, over which we are optimizing, as well as the MOSPA error for $n=2$ (which is the same as a common track accuracy criterion [4]) are given at three points: the mean of the PDF, $\mu$, the most likely hypothesis, $\mu_{M L}$, the point obtained by optimizing over the bound for $n=4, \mu_{4}^{B}$, and the optimal point under the given MOSPA metric, $\mu_{2}^{\text {opt }}$ and $\mu_{4}^{\text {opt }}$.

$$
\begin{aligned}
\mathrm{E}\left[\left(\mathbf{x}^{T} \mathbf{x}\right)^{2}\right] & =\operatorname{tr}[P]^{2}+2 \operatorname{tr}[P P]+2 \operatorname{tr}[P]\left(\mu^{T} \mu\right) \\
& +4 \mu^{T} P \mu+\left(\mu^{T} \mu\right)^{2} \\
\mathrm{E}\left[\left(\mathbf{x}^{T} \mathbf{x}\right)\left(\mathbf{x}^{T} \mathbf{c}\right)\right] & =\left(\operatorname{tr}[P] \mu+2 P \mu+\left(\mu^{T} \mu\right) \mu\right)^{T} \mathbf{c}
\end{aligned}
$$

Designating the $n=4$ MOSPA error bound by $\mu_{4}^{B}$, substituting into (9), we get

$$
\begin{aligned}
N_{T} \mu_{4}^{B} & =k_{0}+k_{1} \hat{\mathbf{x}}_{1}+k_{2}\left(\hat{\mathbf{x}}_{1}^{T} \hat{\mathbf{x}}_{1}\right)+\left(\hat{\mathbf{x}}_{1}^{T} \hat{\mathbf{x}}_{1}\right)\left(k_{3} \hat{\mathbf{x}}_{1}\right) \\
& +\hat{\mathbf{x}}_{1}^{T} k_{4} \hat{\mathbf{x}}_{1}+\left(\hat{\mathbf{x}}_{1}^{T} \hat{\mathbf{x}}_{1}\right)^{2}
\end{aligned}
$$

where

$$
\begin{aligned}
k_{0} & =\operatorname{tr}[P]^{2}+2 \operatorname{tr}[P P]+2 \operatorname{tr}[P]\left(\mu^{T} \mu\right)+4 \mu^{T} P \mu \\
& +\left(\mu^{T} \mu\right)^{2} \\
k_{1} & =-2\left(\operatorname{tr}[P] \mu+2 P \mu+\left(\mu^{T} \mu\right) \mu\right)^{T} A \\
k_{2} & =2\left(\operatorname{tr}[P]+\mu^{T} \mu\right) \\
k_{3} & =-2 \mu^{T} A \\
k_{4} & =4\left(P+\mu \mu^{T}\right) \chi
\end{aligned}
$$

We know that the following gradients are

$$
\begin{aligned}
\nabla_{\mathbf{x}} \mathbf{c}^{T} \mathbf{x} & =\mathbf{c} \\
\nabla_{\mathbf{x}}\left(\mathbf{x}^{T} \mathbf{x}\right)\left(\mathbf{c}^{T} \mathbf{x}\right) & =2\left(\mathbf{c}^{T} \mathbf{x}\right) \mathbf{x}+\left(\mathbf{x}^{T} \mathbf{x}\right) \mathbf{c} \\
\nabla_{\mathbf{x}}\left(\mathbf{x}^{T} C \mathbf{x}\right) & =C \mathbf{x}+C^{T} \mathbf{x} \\
\nabla_{\mathbf{x}}\left(\mathbf{x}^{T} \mathbf{x}\right)^{2} & =4\left(\mathbf{x}^{T} \mathbf{x}\right) \mathbf{x}
\end{aligned}
$$

The gradient of (16) with respect to $\hat{\mathbf{x}}_{1}$ is thus

$$
\begin{aligned}
\nabla_{\hat{\mathbf{x}}_{1}} N_{T} \mu_{4}^{B} & =k_{1}^{T}+2 k_{2} \hat{\mathbf{x}}_{1}+2\left(k_{3} \hat{\mathbf{x}}_{1}\right) \hat{\mathbf{x}}_{1}+\left(\hat{\mathbf{x}}_{1}^{T} \hat{\mathbf{x}}_{1}\right) k_{3}^{T} \\
& +\left(k_{4}+k_{4}^{T}\right) \hat{\mathbf{x}}_{1}+4\left(\hat{\mathbf{x}}_{1}^{T} \hat{\mathbf{x}}_{1}\right) \hat{\mathbf{x}}_{1}
\end{aligned}
$$

The Hessian is

$$
\begin{aligned}
& \nabla_{\hat{\mathbf{x}}_{1}} \nabla_{\hat{\mathbf{x}}_{1}}^{T} N_{T} \mu_{4}^{B}=2 k_{2} \mathbf{I}_{2 d}+2\left(k_{3} \hat{\mathbf{x}}_{1}\right) \mathbf{I}_{2 d}+2 k_{3}^{T} \hat{\mathbf{x}}_{1}^{T} \\
& +2 \hat{\mathbf{x}}_{1} k_{3}+\left(k_{4}+k_{4}^{T}\right)+4\left(\hat{\mathbf{x}}_{1}^{T} \hat{\mathbf{x}}_{1}\right) \mathbf{I}_{2 d}+8 \hat{\mathbf{x}}_{1} \hat{\mathbf{x}}_{1}^{T}
\end{aligned}
$$

Given the objective function, the gradient and the Hessian, we can use an optimization algorithm, such as Newton's method, to approximate the MMOSPA estimate by minimizing the bound. The mean of the normal distribution can be used as an initial estimate.

\subsection{The Solution for a Multivariate Gaussian Mixture Distribution}

If $p(x)$ is a Gaussian mixture, then (8) can be broken down across all of the Gaussians as follows:

$$
\begin{aligned}
& \frac{1}{N_{T}} \int_{\mathbf{x} \in \mathbb{R}^{2 d}}\left\|\mathbf{x}-\hat{\mathbf{x}}_{1}\right\|^{2}\left\|\mathbf{x}-\hat{\mathbf{x}}_{2}\right\|^{2} p(\mathbf{x}) d \mathbf{x}= \\
& \frac{1}{N_{T}} \sum_{i=1}^{N_{H}} \beta_{i} \int_{\mathbf{x} \in \mathbb{R}^{2 d}}\left\|\mathbf{x}-\hat{\mathbf{x}}_{1}\right\|^{2}\left\|\mathbf{x}-\hat{\mathbf{x}}_{2}\right\|^{2} p_{i}(\mathbf{x}) d \mathbf{x}
\end{aligned}
$$

where $p_{i}(\mathbf{x})$ is the PDF of the $i$ th Gaussian, $\beta_{i}$ is its probability, and there are $N_{H}$ Gaussians in the mixture. Thus, the solution is the same as when considering the single Gaussian, except the coefficients of the objective function are replaced with weighted sums of the coefficients of each Gaussian in the mixture.

\section{SIMULATIONS}

We shall evaluate the performance of the estimator minimizing the bound for two one-dimensional targets, $x_{1}$ and $x_{2}$ in two examples ${ }^{7}$. In the first, the mixture consists of two Gaussians with covariance

$$
P_{2}=\left[\begin{array}{cc}
5 & -4 \\
-4 & 5
\end{array}\right]
$$

The first Gaussian has mean $(2,-1.5)$ and the second $(-1.5,2)$. The Gaussians have respective weightings 0.4 and 0.6.

The second example is a Gaussian mixture consisting of 6 components. All Gaussians in the mixture have covariance

$$
P_{6}=\left[\begin{array}{cc}
0.1 & 0 \\
0 & 0.1
\end{array}\right]
$$

The means are $(-1,0),(-1,1),(0,-1),(0,1),(1,-1)$, and $(1,0)$. The weights of the elements in the mixture are respectively $\{45,25,54,45,36,54\} / 259$.

The bound was minimized using Newton's method initialized with the mean of each PDF. The MOSPA errors for $n=4$ and $n=2$ were calculated by discretizing each PDF in a $300 \times 300$ grid of points that was \pm 9 and \pm 2 about the mean respectively for the the two Gaussian, and the six Gaussian mixtures. The optimal MOSPA estimates for $n=2$ and

\footnotetext{
${ }^{7}$ Space constraints keep us from considering more complex scenarios.
} 


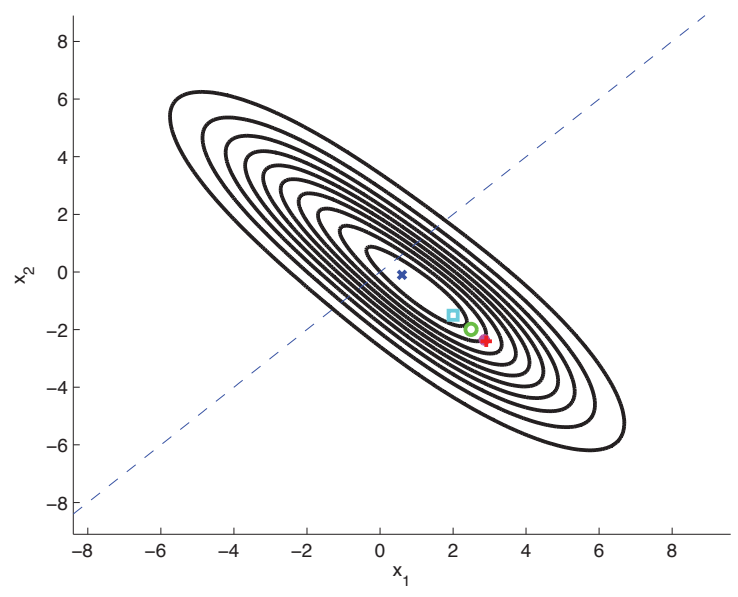

(a) 2 Gaussian Mixture PDF

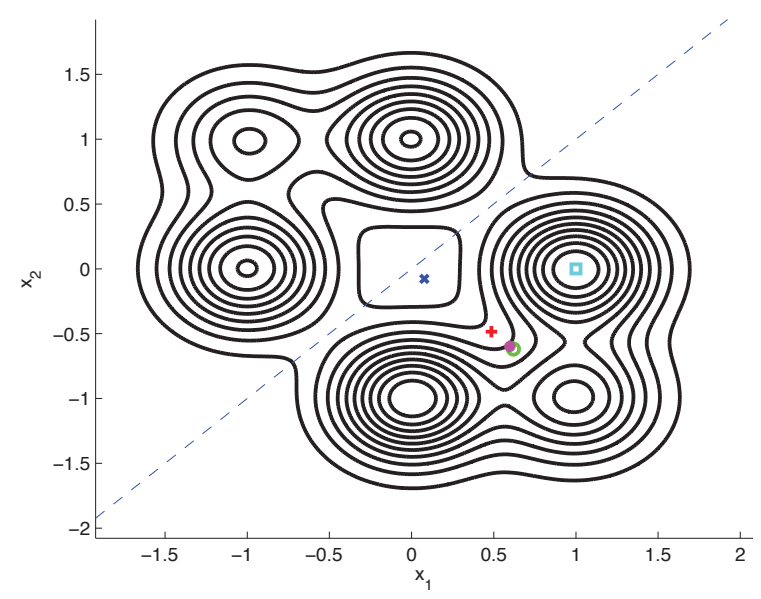

(b) 6 Gaussian Mixture PDF

Fig. 1. The PDFs. The dashed lines represent where $x_{1}=x_{2}$. The ' $\mathrm{x}$ ' marks the mean of the PDF, the square the mean of the most likely hypothesis, the plus-sign the estimate obtained by minimizing the bound, the 'o' the MOSPA optimal estimate for $n=2$ and the filled circle the MOSPA optimal estimate for $n=4$. In (a), the optimization estimate and the $n=4$ MOSPA estimate markers overlap. In (b) the two MOSPA markers overlap.

$n=4$ were determined by evaluating the error at every point on the $300 \times 300$ grid of points of the discretized PDF.

The MOSPA error, under two different MOSPA metrics is shown in Table 1. The PDFs of the mixtures are shown in Figure 1 along with the location of the mean, $\mu$, the peak of the most likely Gaussian in the mixture $\mu_{M L}$, the point obtained from the bound optimization $\mu_{4}^{B}$ and the optimal MOSPA estimates for $n=2, \mu_{2}^{\mathrm{opt}}$, and for $n=4, \mu_{4}^{\mathrm{opt}}$. The approximate minimum MOSPA estimate outperformed using the overall mean or the mean of the most likely hypothesis.

\section{CONCLUSIONS}

We derived an upper bound on the MOSPA error when using the $l_{2}$-norm raised to the fourth as the distance measure. This approximation allows us to eliminate the integral needed to evaluate the MOSPA error when the moment generating function for the multivariate PDF in question can be explicitly written. We demonstrated that minimizing the bound can improve the MOSPA error of the estimates beyond simply using the MMSE or the ML hypothesis. This give us the "smoothing" effect of an MMSE estimate without the coalescence problems that come when track identity is lost.

\section{REFERENCES}

[1] Y. Bar-Shalom, X. R. Li, and T. Kirubarajan, Estimation with Applications to Tracking and Navigation. New York: John Wiley \& Sohns Inc., 2001, ch. 1.4.15.

[2] I. P. Bottlik and S. S. Blackman, "Coordinated presen- tation of multiple hypotheses in multitarget tracking," in Proceedings of SPIE: Signal and Data Processing of Small Targets Conference, vol. 1096, Sep. 1989, pp. 152159.

[3] D. F. Crouse, Y. Bar-Shalom, P. Willett, and L. Svensson, "The JPDAF in practical systems: Computation and snake oil," in Proceedings of SPIE: Signal and Data Processing of Small Targets Conference, vol. 7698, Apr. 2010.

[4] B. E. Fridling and O. E. Drummond, "Performance evaluation methods for multiple target tracking algorithms," in Proceedings of SPIE: Signal and Data Processing of Small Targets Conference, vol. 1481, Aug. 1991, pp. 371-383.

[5] M. Guerriero, L. Svensson, D. Svensson, and P. Willett, "Shooting two birds with "two" bullets: How to find minimum mean OSPA estimates," in Proceedings of the 13th International Conference of Information Fusion, Edinburgh, Scotland, Jul. 2010.

[6] K. Pattipati, T. Kirubarajan, and R. L. Popp, "Survey of assignment techniques for multitarget tracking," in Multisensor-Multitarget Tracking: Applications and Advances, Y. Bar-Shalom and W. D. Blair, Eds. Boston, MA: Artech House, 2000, vol. 3.

[7] D. Schuhmacher, B.-T. Vo, and B.-N. Vo, "A consistent metric for performance evaluation of multi-object filters," IEEE Transactions on Signal Processing, vol. 56, no. 8, pp. 3447-3457, Aug. 2008. 